\title{
Two Kinds of Universals and two Kinds of Groups
}

\author{
Friederike Moltmann \\ University of Liverpool
}

Philosophers have long debated about two ways of conceiving of universals: as Platonic universals and as Aristotelian universals. Roughly, Aristotelian universals are inherent in the particulars that instantiate them; they can be multiply located (located just where the instances are located), and they exist only if they have at least one instance. Platonic universals, by contrast, are truly abstract objects: their existence is independent of the particulars that instantiate them, and they are not located in space and time at all. This historical distinction between two conceptions of universals, I would like to suggest, can be construed in terms of two ways of assigning properties to entities: Aristotelian universals are assigned properties only on the basis of properties of or relations among their instances (e.g. an Aristotelian universal has a locational property $\mathrm{P}$ just in case some instances of that universal has P). By contrast, platonic universals are assigned properties just like ordinary objects (Platonic universals thus can't be located in space and time because there is no particular from which they could inherit a location). If the distinction between Aristotelian and Platonic universals is conceived in this way, then the view becomes plausible that both sorts of universals can exist simultaneously.

In this paper, I will argue that a distinction in the way of assigning properties to entities plays an important role in the semantics of natural language, namely first in the semantics of nominalizations that refer to universals (wisdom, the property of being wise), and second, in an analogous way, in the semantics of plurals. Natural language, I will argue, allows reference to two kinds of universals and two kinds of groups which are distinguished from each other by the way their properties are fixed: namely either in the ordinary way (as with individuals) or on the basis of properties of or relations among instances or group members - in particular by projecting properties of instances or group members onto the universal or group itself. It is this distinction, I will argue, rather than some formal distinction among two sorts of entities, that helps explain some crucial facts about nominalizations and plurals, such as different readings of certain classes of predicates, the way existential constructions and intensional predicates are understood, and the possibility of distributive interpretation.

\section{Basic assumptions}

First some important basic assumptions. I will assume a Neocarlsonian acc ount on which bare plurals and mass nouns generally stand for kinds, triggering an existential reading of episodic predicates (1) and a universal or generic reading of individual-level predicates (2) (cf. Carlson 1977, Chierchia 1998):
(1) a. John found gold.
b. John bought apples.
a. Gold is shiny. 
b. Apples are healthy.

Moreover, bare mass nouns and plurals are acceptable with intensional verbs like need. With such verbs, as Chierchia (1998) (citing Carlson 1977) emphasizes, they only allow an intensional reading:

(3) a. John needs gold.

b. John needs apples.

The intensional reading that need in ( $3 \mathrm{a}, \mathrm{b}$ ) displays can be paraphrased in terms of quantification over possible objects as follows: (3a) says that John's needs are satisfied only if he has some quantity of gold, and (3b) that they are satisfied only if he has some quantity of apples. (4):

Bare mass nouns and plurals can occur also in existential constructions, as in

(4) a. Gold exists.

b. Unicorns don't exist.

Existential constructions with bare plurals and mass nouns claim the existence of instances and not, as one might have thought, the existence of the kind independently of the instances.

Bare plurals and mass nouns finally allow for what is generally considered genuine kind predicates such as extinct, rare, and widespread:

(5) a. Dinosaurs are extinct.

b. Pink diamonds are rare.

c. Pidgins are widespread in Europe.

What is characteristic about these predicates is that they measure in some way the distribution of the instances of the kind, possibly across different times and different actual and counterfactual situations. Let me therefore call those predicates instance distribution predicates.

A particularly strong evidence, not previously noted, that bare plurals and mass nouns always stand for kinds is that they allow the different kinds of predicates simultaneously, as in (6):

(6) a. Pink diamonds are rare, hard to get, and very expensive.

b. John needs something that is rare, hard to get, and very expensive, namely pink diamonds.

In (6a), a bare plural is to fulfill a conjunction of predicates with conjuncts that are instance-distribution predicates, episodic predicates, and individual-level predicates. (6b) shows the same with something as a quantifier ranging over kinds.

Such examples make clear that the readings of different kinds of predicates with bare plurals or mass nouns must be a matter of the interpretation of the predicate, rather than the interpretation of the bare NP. 
The Neocarlsonian account of bare plurals and mass nouns can naturally be extended from simple, underived nouns to nominalizations. There are various kinds of nominalizations all of which, it appears, trigger the same readings of predicates when occurring without determiner.

First, there are deverbal nominalizations that stand for kinds of events or kinds of states, for example laughter: In (7a) an episodic predicate triggers existential quantification over laughing events; in (7b) an individual-level predicate triggers generic quantification; (7c) displays the relevant readings with an intensional verb, (8d) with an existential construction; and (7e) contains an instance-diostribution predicate:

(7) a. John heard laughter.

b. John likes laughter.

c. John longs for laughter.

d. There is laughter everywhere.

e. Genuine laughter is rare.

Second, there are nominalizations of adjectives such as beauty, wisdom or generosity. Such nominalizations, which will be discussed in greater detail in the next section, do not stand for kinds of states, but rather for kinds of particularized properties or what philosophers nowadays call 'tropes' (cf. Stout 1952, Williams, 1954, Simons 1994, Lowe 1998). That is, the instances of beauty are things like Mary's beauty or the painting's beauty, and it is this, the particular instantiation of beauty, that is admired, described, or compared in (8), rather than the state of being beautiful:

(8) a. John admired Mary's beauty.

b. John described Mary's beauty.

c. Mary's beauty exceeds Sue's beauty.

In (8a-c), Mary's beauty cannot be replaced by Mary's being beautiful (referring to Mary's state of being beautiful). Intutively, this is because tropes 'focus' on the way a particular property is instantiated, whereas states 'focus' on the mere fact that the property is instantiated. The present task, however, is not to give an account of the difference between events, states, and tropes, but to make clear that bare underived and derived nouns behave exactly alike with respect to the relevant classes of predicates and thus should all be considered kind-referring terms - whether the instances of the kind are individuals, quantities, events, states, or tropes. In what follows, let us focus on nominalizations referring to kinds of tropes.

\section{3. the distinction among two sorts of universals}

There are two ways of referring to universals: one is by using the term the property of being .... for example the property of being generous, let's call those 'explicit property-referring terms', the other is by using a bare adjective nominalization, for example generosity. These two ways of referring to universals are not equivalent, however, but rather trigger fundamentally different readings of various kinds of 
predicates. Five kinds of predicates can be distinguished that display different readings or degrees of acceptability with explicit property-referring terms and bare nominalizations

i. episodic predicates

(9) a. I have encountered hostility.

b. I have encountered the property of being hostile.

(10) a. Generosity is rarely reciprocated.

b. The property of being generous is rarely reciprocated.

Whereas (9a) is true if I have encountered an instance of hostility, (9b) can only mean that I have never encountered the abstract object (the property of being hostile). Similarly for (10), where (10b), unlike (10a), does not make sense in the first place.

ii. predicates of evaluation

(11) a. Friendliness is nice.

b. The property of being friendly is nice.

(12) a. Ordinariness is boring.

b. The property of being ordinary is boring.

(13) a. Originality is interesting.

b. The property of being original is interesting.

Whereas the application of nice to friendliness in (11a) must be based on the evaluation of instances of friendliness (friendly people, gestures, behavior) being nice, nice in (11b) evaluates the abstract object (implying e.g. that it has nice formal properties) and cannot be understood as evaluating the instances. Similarly for (12) and (13).

iii. intensional predicates

(14) a. John is looking for honesty.

b. John is looking for the property of being honest.

(15) a. John needs efficiency.

b. John needs the property of being efficient.

Whereas John's search according to (14a) is satisfied if John finds an instance of honesty, the satisfaction of his search in (14b) requires him to find the abstract object. (14a) displays only what looks like an intensional reading, but (14b) naturally has an existential reading, presupposing the existence of the abstract object. Similarly for (15).

iv. existential constructions

(16) a. Generosity exists.

b. The property of being generous exists.

(16a) is true just in case there is an instances of generosity; by contrast, (16b) is true just in case the abstract object as such exists, regardless of its instantiations. 
v. instance distribution predicates

(17) a. Honesty is rare.

b. Sloppiness is widespread.

(18) a. ?? The property of being honest is rare.

b. ?? The property of being sloppy is widespread.

(17) shows that instance-distribution predicates are perfectly acceptable with bare adjective nominalizations. By contrast, many speakers dislike them with explicit property-referring terms as in (18). 1

Generally, these data show that whereas predicates apply as with ordinary individuals in the case of explicit property-referring terms, they apply with bare nominalizations only by in some way targeting the instances first and only derivatively the kind. Thus, an episodic predicate applies to a kind on the basis of some instance fulfilling the property that is the 'literal meaning' of the predicate; an evaluative (that is, individual-level) predicate applies to a kind on the basis of all instances (or rather suitable, typical instances under suitable circumstances) fulfilling the property that is the 'literal meaning' of the predicate. An intensional predicate applies to a kind with the kind of meaning the predicate has when it acts as an intensional verb taking an indefinite NP complement. The existential verb exist applies to a kind in the way it would apply to an individual concept, claiming a nonempty extension at the relevant index. Finally, instance-distribution predicates semantically select only kinds, not properties.

Explicit property-referring terms and bare nominalizations thus differ with respect to the basis for the application of a predicate, in the reading a predicate displays, as well as in the acceptability of a predicate. How should one account for these differences?

One way might be by separating the content of a predicate from the way a predicate says something about an argument - that is, to not identify the 'attribution' of the predicate to an argument with the ascription of the property expressed by the predicate. For example, the predicate is reciprocated in (10a) would be attributed to the kind 'generosity' not because the kind has the property expressed by the predicate, but because some instance fulfills it; and nice in (11a) would be attributed to the kind 'friendliness' not because the kind is nice, but because the instances of the kind generally are.

This strategy works, however, only for the first two kinds of predicates (i and ii). It is not applicable to the others (iii-v): when an intensional predicate takes a kind as argument, it fails to display an extensional reading, and in fact the extensional meaning cannot be taken to be fulfilled by instances of the kind. Moreover, exist when it applies to a kind does not apply to any particular instance in the way it would with a definite NP. Finally, the acceptance and resistance of instancedistribution predicates by explicit property-referring terms and by bare nominalization cannot be explained by reinterpreting the semantic relation between predicate and argument.

I will pursue an account that rests on a fundamental distinction between semantic structure on the one hand and ontological or metaphysical structure on the other hand and a corresponding distinction among two sorts of entities. The semantic structure of a sentence imposes one notion of an entity, namely an entity is whatever can be referred to by a referential NP (or singular term) (Frege) or act as 
the value of a variable (Quine). I will call such entities semantic objects. The notion of a semantic object, however, is to be contrasted with that of an ontological object.

Objects in the ontological sense are not characterized in terms of any linguistic criterion, but rather in terms of purely metaphysicial or ontological conditions. Both kinds and properties are semantic objects. But only properties are ontological objects. I will also say, more simply, that properties are objects, whereas kinds are nonobjects.

The metaphysical criterion that distinguishes objects from nonobjects is not, as one might expect, having particular identity and existence conditions (both properties and kinds may or may not do) or having a boundary. Rather objects in the ontological sense are those entities that can fulfill what I call basic properties. Nonobjects, by contrast, are objects that can fulfill nonbasic or derived properties. Objects, moreover, cannot fulfil derived properties, and nonobjects cannot fulfil basic properties.

Basic properties are the kinds of (simple or complex) properties we are best familiar with: being encountered by me, being located at a particular place, being nice, and being interesting are basic properties. Being rare, widespread, extinct, by contrast, are not: they are properties that are to be understood on the basis of basic properties or relations, such as the property of existing at time $t$ at place l. Roughly, basic properties (and relations) are properties not reducible to properties of or relations among lower-order entities. Thus, whereas 'being interesting' is a property that evaluates an entity as a whole, 'being rare' is a property of kinds obviously reducible to relations among instances (and numbers measuring instances). A kind is rare in case, roughly, the spatial and/or temporal distance among the (spatially and/or temporally) closest instances is greater than expected (or greater than some given norm).

In first approximation, the notions of a basic and a non-basic or derived property can be given as follows:

(19) a. A property $\mathrm{P}$ is a basic property iff it cannot be reduced to properties of or relations among lower-order entities (for instance if $\mathrm{P}$ is a property of kinds).

b. A property is a derived property iff it is not a basic property.

With the help of such a (as yet to be further explained) notion of basic property, we can give the following definition of an object:

(20) a. An entity d is an object iff for some basic property $P, P(d)$ or not-P(d) and for no derived property $\mathrm{Q}, \mathrm{Q}(\mathrm{d})$ or non-Q(d).

b. An entity $\mathrm{d}$ is a nonobject iff for no basic property $\mathrm{P}, \mathrm{P}(\mathrm{d})$ or not-P(d) and for some derived property $\mathrm{Q}, \mathrm{Q}(\mathrm{d})$ or non-Q(d).

Thus, objects are entities of which a given basic property may be either true or false, whereas nonobjects are entities of which a basic property is never true nor false.

Properties differ from kinds simply in that they are objects in the sense of being potential bearers of basic properties. Kinds cannot fulfill basic properties, which is why predicates like is reciprocated and nice cannot be predicated of kinds by claiming that the kind is literally reciprocated or nice. However, predicates like is 
reciprocated and nice can be predicated of kinds. But when they are predicated of kinds, then, I want to argue, they have an extended meaning, namely a meaning on which the property of being reciprocated or the property of being nice is to be fulfilled only by instances of the kind in order for the predicate to hold.

A predicate that expresses a basic property (i.e. not an instance-distribution predicate) applies to a property with its literal meaning, requiring the property as an abstract object to fulfil the basic property. But when such a predicate applies to a kind, it will apply with an extended meaning, a meaning that is now a derived property, construed on the basis of the basic property. For example, the derived property of being reciprocated holds of a kind just in case some instance of the kind fulfils the basic property of being reciprocated, and the derived property of being nice holds of a kind just in case generally any instance fulfils the basic property of being nice. The extended meaning of an episodic predicate whose original meaning was a property $\mathrm{P}$ will be $\lambda \mathrm{x}\left[\exists \mathrm{x}\left(\mathrm{x}^{\prime} \mathrm{Ix} \& \mathrm{P}\left(\mathrm{x}^{\prime}\right)\right)\right]$, where $\mathrm{I}$ is the instantiation relation. The extended meaning of an individual-level predicate whose original meaning was $\mathrm{P}$ will, roughly, be $\lambda x\left[\mathrm{Gn} \mathrm{x}^{\prime}\left(\mathrm{x}^{\prime} \mathrm{Ix}-->\mathrm{P}\left(\mathrm{x}^{\prime}\right)\right)\right]$, where ' $\mathrm{Gn}$ ' is a generic quantifier.

The readings the predicates in (iii-v) display when applying to kinds require yet other kinds of extended meanings. Concerning intensional predicate taking kinds as arguments as in (15a) and (16a), first recall that they display only an intensional reading. That is, (15a) could not possible mean 'there is an instance of honesty $\mathrm{x}$ and John is looking for $\mathrm{x}$ '. I take this to mean the following: when look for takes a kind as an argument, it is in fact extensional, just as when it takes an individual as argument (John is looking for Mary). The apparent intensional reading arises because with kind arguments look for has an extended meaning which is based on the literal meaning look for has when it occurs intensionally rather than extensionally. The 'extension' of the meaning of lookfor to kind arguments consists in understanding the look for-relation as a relation between individuals and kinds in the way the look for-relation in the intensional sense is understood when it takes properties (or intensional quantifiers) as arguments. ${ }^{2}$ Thus, look for with an extended meaning, look for ext, can be defined as follows, where look forint is the intensional look for-relation and int is the function mapping a kind onto its intension - that is, the function mapping a world $w$ and time $t$ onto the set of instances of the kind at $w$ and $t$ :

(21) For an object $d$ and a kind $k$, look forext $(d, k)$ iff look forint $(d$, int(k))

Let us turn to exist as in (iv). Exist when taking a kind as an argument can be given an extended meaning existext on the basis of its literal meaning as follows:

$$
\text { exist }_{\text {ext }}(\mathrm{k}) \text { iff exist(int(k)). }
$$

That is, exist in the extended sense applies to a kind on the basis of its literal meaning applying, in the familiar way, to the intension associated with the kind. The literal meaning of exist is of course the one we find in the kind exists, where, following common assumptions, the kind stands for the intension of the kind, an individual concept (a function from world-time pairs to individuals). 
Given the four ways of extending predicate meanings for kind arguments, the question arises: what is the status of such predicate extensions? Should they constitute separate predicates or a disjunct of a a broadened disjunctive lexical meaning of the same predicate?

In the case of kind predicates, there is strong evidence for the latter: a question such as (23a) can be answered either by (23b) (mentioning an object) or (23c) (mentioning a kind), and thus the occurrence of buy in (23a) should include both the object-oriented and the kind-oriented meaning:
a. What did John buy?
b. Apples.
c. My painting.

Thus, I will assume that the general meaning of a predicate is a disjunction consisting of a basic property as one disjunct (the 'literal meaning') and a suitable extension for kinds (in one of the four ways of extending meanings). There is one exception to this, however, and that is instance-distribution predicates.

Instance-distribution predicates as in (17) cannot be handled in terms of predicate extensions. What goes on with instance-distribution predicates rather is this: instance-distribution predicates inherently have a meaning which is derived from basic properties holding of objects; they do not express basic properties in the first place - as they are inapplicable to objects such as properties. The property expressed by widespread, for example can be described roughly as follows: it is a property that holds of a kind $\mathrm{k}$ iff for a sufficient number of regions $\mathrm{r}$, there are instances $i$ such that $i$ is located at $r$. In this way, widespread expresses a derived, nonbasic property, based on the basic relation of 'being located at'.

Derived properties that can be fulfilled by kinds thus need not always constitute a derived lexical meaning of a predicate, but may be the basic lexical meaning of a predicate (they are in this case derived properties, but basic, rather than derived meanings). Again, this shows a discrepancy between metaphysical structure and semantic (lexical) structure.

Let me conclude the discussion of NPs referring to universals with some remarks concerning the formation and compositional semantics of explicit propertyreferring terms. Explicit property-referring terms are generally obtained syntactically from a reifying noun (e.g. property) and a kind-referring NP complement that refers either to a kind of trope (as in the property of honesty) or to a kind of states (as in the property of being honest). Semantically, this means that the expression the property of (or for that matter the attribute of, the virtue of, or the quality of) acts as a reifying expression mapping a nonobject, a kind, onto the corresponding object. Thus, the semantics of property (of) when taking a complement will be roughly as follows:

(24) For a kind-referring term $\mathrm{X}$, [property of $\mathrm{X}]=[$ property of $]([\mathrm{X}])$ $=\{\operatorname{reif}([\mathrm{X}])\}$, where $\operatorname{reif}([\mathrm{X}])$ is the entity o such that o is like $[\mathrm{X}]$, except that $o$ is an object .

There is one class of exceptions to the generalization that (basic) properties cannot be attributed to kinds. These are sortal predicates with head nouns like virtue, 
attribute, or property. (25) shows that such predicates allow for kind-referring terms as subjects:
a. Friendliness is my favorite attribute.
b. Courage is an admirable property.
c. Honesty is a virtue.

There are several possible ways of accounting for such cases.

First, nouns like virtue. attribute, and property might have an extended meaning on which they also apply to kinds.

One problem with this proposal is that property should now be able to occur without its reifying force when taking a complement, which it cannot. Another problem is that further attributes modifying the predicate have the reading on which they apply to an abstract object, not a kind, for example interesting in (26):

\section{Ordinariness is a property that is interesting.}

Second, the copula might be the is of constitution, rather than the is of predication: a kind of trope, after all, in a way constitutes a property.

This account might work for (25a), but it won't work not for $(25 b, c)$. For the account to work for (25b), the NP an admirable property would have to be understood as a quantificational, not a predicative NP. That is, (25b) would be interpreted as: for some admirable property $\mathrm{P}$, courage constitutes $\mathrm{P}$. But then the problem arises that when the sentence is negated as below in (27a), an admirable property cannot take scope over the negation, with the reading in (27b):

a. Courage is not an admirable property.

b. For an admirable property $\mathrm{P}$, courage is $\mathrm{P}$.

A third alternative is to take sortal predicates to trigger a slight reference shift in the subject, triggering a switch from referring to a kind to referring to the reification of the kind, the property. Thus, (25b) would be analysed as in (28):

$$
\text { admirable property (reif([courage }]))
$$

Thus, courage in (25b) would be a 'concealed property', to use Grimshaw's (1979) terminology.

There are several difficulties arising for this proposal. First, on the analysis in (28) it would be quite strange why other predicates, such as interesting, do not allow for the same reference shift for its subject. Second, it is implausible that such reference shift takes place in the first place. Thus, a nonrestrictive relative clauses as in (29a) requires the head noun to still refer to a kind, rather than a property, which makes it hard to reconcile with the alleged reference shift triggered by the predicate. Similarly for (29b), where the pronoun must refer back to a kind, rather than a property::

(29) a. True courage, which one sees only rarely, is an admirable property.

b. True courage is an admirable property, even though one sees it only 
rarely.

The fourth altemative, which I will endorse, assimilates (25b) to the construction in (30):

the property of courage

The expression the property of, given (24), expresses the function of reification reif, applying to kinds of tropes. Reification, on this analysis, is also involved in (25b), which will be analysed as 'courage is something such that when reification applies to it, the result is an object that is admirable'. More formally:

(31) $[$ is an admirable property $]=\lambda \mathrm{x}[\operatorname{admirable}([$ property of $](\mathrm{x}))]=$ $\lambda x[\operatorname{admirable}(\operatorname{reif}(\mathrm{x})))]$

\section{Plurals}

\section{1. pluralities and groups}

As is well-known, there are two ways of referring to group-like entities: with a plural $\mathrm{NP}$ as in (32a) and with a collective NP, as in (32b):

(32) a. The children are asleep.

b. The group of children is asleep.

Let me call the kind of entity referred to by a plural a plurality and the kind of object referred to by a collective NP a group.

I follow the tradition of Link (1983) and others according to which definite plural and singular NPs have an analogous semantics: the child stands for the only child in the context, the children stands for the maximal group of children in the context. $^{3}$

In the majority of cases when a plural NP refers to a plurality composed of the same individuals as the group referred to by a collective NP, the plurality and the group seem to count as distinct entities. Thus, the referent of the orchestra has different identity and existence conditions from the referent of the members of the orchestra. If members of the orchestra are replaced, the orchestra may remain the same, but not what would be referred to as the orchestra members, and the orchestra members may still exist even if the orchestra has ceased to be. The difference shows up also in the way the things are counted. There is one orchestra, but several orchestra members. Referents of collective NPs, groups, generally are counted as one, but referents of plurals, pluralities as many.

In what follows, I will argue that the underlying difference between pluralities and groups is exactly the same as what distinguishes kinds from properties: pluralities pattem together with Aristotelian universals, whereas properties pattern together with Platonic universals; that is, pluralities have their properties fixed on the 
basis of properties of or relations among group members, whereas groups have their properties fixed in the ordinary way. 4

\section{2. analogous facts}

The crucial observations are that plural and collective NPs differ in ways strikingly similar to the ways bare nominalizations and explicit property-referring terms differ from each other, that is, with respect to their behavior with different kinds of predicates.

These are the classes of predicates or readings of predicates with which plurals and collective NPs behave differently:

i. distributivity

Generally only definite plurals, not definite collective NPs allow for distributive interpretations of predicates (that is, of predicates that would allow both a collective and a distributive interpretation) (cf. Moltmann 1997a):

(33) a. The things are heavy.

b. The collection of things is heavy.

a. The team lifted the piano.

b. The team members lifted the piano.

(33a) and (34a) allow for both a collective and a distributive interpretation, whereas (33b) and (34b) allow only for a collective interpretation.

2. predicates making reference to group members

Collective predicates whose content makes reference to group members, but not to the group as a whole, allow only for plural NPs as complement, not for collective NPs. These are predicates such as compare, distinguish (w.r.t. the object argument position), like each other, and similar, whose content is based on binary relations among group members. They also include predicates like count or numerous, whose content is based on a function applied to all the group members. The generalization is what I in Moltmann (1997) called the 'Accessibility Requirement':

(35) a. John compared the students.

b. \# John compared the class.

(36) a. The students like each other.

b. \# The class likes each other.

(37) a. John cannot distinguish the students.

b. \# John cannot distinguish the class.

(38) a. The students are similar.

b. \# The class is similar.

(39) a. John counts the students.

b. John counted the group of students. (means: he counted one)

a. The students are numerous.

b. \# The class is numerous.

3. predicates of existence

With definite plurals, the verb exist can claim only the existence of group members. It cannot, unlike with collective NPs, claim the existence of the group as such (for 
example as a reply to a sceptic concerning the existence of groups, sums, etc. as entities above individuals):

(41) a. The students do not exist.

b. The class does not exists.

c. The sum / collection / group of students does not exists.

(41a) denies the existence of the individual students; (41b) denies the existence of the class, independently of the existence of the students; as does (41c) with respect to entities like sums, collections, or groups.

It should be obvious what approach I will take to explain the difference in behavior of plural and collective NPs. Pluralities are nonobjects, that is, they cannot have basic properties, but only derived ones. Groups, by contrast, are objects and thus can fulfil basic properties, but not derived ones. For pluralities, the characterization of basic and derived property as in (19) needs to be extended, of course, so that basic properties are also those that are not reducible to properties of or relations among group members. The notion of basic property then becomes somewhat more problematic, however. Many properties may be reducible to properties of or relations among group members, without counting as a derived property for current purposes, for example even configurational properties or properties of size, as discussed below. The proper way of understanding the notion of basic and derived property therefore should be as follows. Basic properties are primitives and a derived property is a property constructed from basic properties either by one of the (rather limited) ways of extending predicate meanings or else by the particular way the meaning of instance distribution predicates is constructed from locational and measurement properties.

The operation of sum formation involved in the semantics of plurals will now always map a set of more than one individual or sum onto a nonobject. Thus, if the domain of entities $\mathbf{E}$ divides into two subdomains, the domain $\mathbf{O}$ of objects and the domain $\mathbf{N}$ of nonobjects, we will have the following conditions on the operation sum, which will apply in the case of definite plurals as in (42b):
a. For a nonempty set $\mathrm{X} \subseteq \mathrm{E},|\mathrm{X}|>1, \operatorname{sum}(\mathrm{X}) \in \mathrm{N}$.
b. $[$ the children $]=\operatorname{sum}([$ children $])$

The expression group of, when followed by a definite plural (e.g. the group of the children) will map a plurality onto the corresponding group, by the same operation of reification reif used in the case of kinds:

(43) For an entity $\mathrm{d} \in \mathbf{N}$, [ group of $](\mathrm{d})=\operatorname{reif}(\mathrm{d})=$ the object o that is just like $\mathrm{d}$ except that $o \in \mathbf{O}$.

When followed by a bare plural (the group of children), reif maps a kind onto the reification of the sum of its extension at the relevant world and time $(\operatorname{int}(k)(w, t))$ :

(44) For a kind $\mathrm{k}$, [group of $](\mathrm{k})=\operatorname{reif}(\mathrm{k})=$ the object o that is just like $\operatorname{sum}(\operatorname{int}(k)(w, t))$ except that $o \in \mathbf{O}$. 
My account of predicates that apply to the two kinds of universals cannot simply be carried over to groups and pluralities. The sorts of meaning extensions with which predicates can apply to kinds do not as such apply to pluralities. It is necessary therefore to go in detail through the different kinds of predicates and the way they can apply to groups or pluralities.

First distributivity. Obviously, distributive interpretation consists in applying a predicate to a plurality on the basis of all members of the plurality fulfilling the literal meaning of the predicate (a basic property) - just as in the case of applying individual-level predicates to kinds. However, the facts are not as straightforward as in the case of predicates taking kinds as arguments. In analogy with how individuallevel predicates apply to kinds, we would expect predicates expressing basic properties to apply to a plurality only in a distributive way. However, this expectation is not borne out. Pluralities allow for a wide range of predicates with collective interpretations (other than those in (ii)). These are some rather familiar examples:

(45) a. The children surrounded the palace.

b. The men lifted the piano.

(46) a. The stones weigh 10 kilo.

b. The picures take up a lot of space.

The examples in (45-46) display both distributive and collective readings.

On the other hand, there are also predicates that cannot apply to pluralities in a collective way, even though their content should in principle be applicable in the same way as with groups:

(47) a. The children are big. (no collective reading)

b. The group of children is big. (collective reading)

(48) a. The pictures are large. (no collective reading)

b. The collection of pictures is large. (collective reading)

The different behavior of the predicates in (45-46) from the one of those in (47-48) must reside in their particular descriptive content. What is crucial about the predicates in (45) seems to be that they describe the participation of a plurality in an event, whereas the predicates in (47-48) don't. In order for (45a) and (45b) to be true, a significant number of the group members each will have to contribute to the causation of the event described by the predicate.

That participation in an event is the crucial parameter is also supported by the way evaluative predicates apply to pluralities and groups:

(49) a. The children are impressive. (given what they do, how they interact)

b. The group of children is impressive. (given its configuration, composition)

Impressive can have a collective interpretation with plurals, as in (49a) but it can then evaluate only the collective participation of the group members in an event, not the composition or configuration of the group, as is possible with collective NPs as in (49b). 
Somewhat different are the predicates in (46). Here the predicate describes a cumulative relation of the group members to a measure (10 kilo) or space ('a lot of space').

Thus, the characteristic of predicates allowing for a collective interpretation with plurals appears to be that the predicate relates the group to another entity to which the individual group members are each related in a particular way. On such a collective interpretation, the predicate can then be considered as having a derived property as its content, a property reducible to basic relations relating the individual group members to another entity. In this case, we have a reanalysis of a basic property as a derived property.

Turning now to the formalization of distributivity, the extended distributive meaning of a predicate $\mathrm{N}$ will be as in (50):

$$
\left[\mathrm{N}_{\text {distr }}\right]=\left\{\mathrm{x} \mid \forall \mathrm{x}^{\prime}\left(\mathrm{x}^{\prime} \mathrm{P} \mathrm{x}-\mathrm{N}\left(\mathrm{x}^{\prime}\right)\right)\right\}
$$

Again we must ask the question: what is the formal status of this extension?

There is reason not to assume that it constitutes an additional disjunct in a disjunctive meaning of the predicate. That is because when a speaker utters a sentence like (51), he can't leave it open whether the predicate is to be understood distributively or collectively. Rather he must have one or the other reading in mind:

The students lifted the box.

Distributivity thus is better seen as generating a separate lexical meaning. For every predicate $\mathrm{N}$, there will then be a related predicate $\mathrm{N}_{\text {distr }}$, which is the distributive extension of $\mathrm{N}$ for its application to puralities.

The predicates in (35-40) clearly correspond to the class of instancedistribution predicates with kinds. They have a content that is obviously obtained from basic relations to be fulfilled by the individual group members. Thus, their content is a derived property, a property that can be fulfilled only by nonobjects like pluralities and not by objects such as groups.

Let us turn to the predicate exist as in (41), which with pluralities cannot claim the existence of the collection as such, but only the existence of the members of the collection. Here the analogy to kinds does not quite hold: exist when applied to kinds, I suggested, gets an extended meaning on which its application to the kind is equivalent to the original meaning of exist applying to the intension corresponding to the kind (the function from indices to sets of instances). In the case of pluralities, exist appears to apply distributively to the intension of each individual group member. Thus, (41a) will be analysed as in (52) with the extended distributive meaning of exist: (Here < is the relation between group members and pluralities.)

$$
\neg \operatorname{exist}_{\operatorname{distr}}(\operatorname{sum}(\{\operatorname{indiv}-\operatorname{conc}(\mathrm{x}) \mid \mathrm{x}<[\text { the students }]\})
$$

No account of plurals is complete without dealing with and when applying to two pluralities. As is well-known, and either generates a mixed group consisting of the members of the two pluralities or a higher-order group having the two pluralities as members: 
John compared the dogs and the cats.

(53) can either mean that John compared individual dogs and cats or that he compared the dogs to the cats. On the present account, the two readings of and are best dealt with by assigning it two different meanings. The first meaning, forming mixed groups, will be as in (54):

$$
\left[a n d_{\mathrm{S}}\right]\left(\mathrm{d}, \mathrm{d}^{\prime}\right)=\operatorname{sum}\left(\left\{\mathrm{d}, \mathrm{d}^{\prime}\right\}\right)
$$

The second meaning will first map the two pluralities onto corresponding objects before forming a group with two members:

$$
[\operatorname{and} 2]\left(d, d^{\prime}\right)=\operatorname{sum}\left(\operatorname{reif}(d), \operatorname{reif}\left(d^{\prime}\right)\right)
$$

\section{3. other accounts of plurals}

On the present account, the two kinds of groups and the two kinds of universals share fundamental similarities and are distinguished by the same underlying parameter concerning the fulfillment of properties. The focus of this account is the nature of properties: entities are distinguished by what properties they can fulfil and the acceptability and the readings of predicates is explained in terms of the nature of the properties they express. With its focus on properties, the account differs fundamentally from the existing accounts that assume a uniform semantics of singular and plural definite NPs, which generally focus on the inherent nature of the entities referred to. In what follows, I will briefly discuss these accounts to see how they would fare in explaining the data in question.

\subsection{1. positing different formal objects}

A very influential account in the semantic literature has been the lattice-theoretic account of Link (1983). For Link, pluralities come form a domain that is ordered by a transitive part of-relation and whose atoms are individuals. Formally, this is a joint semilattice with atoms $\angle \mathrm{E},<\mathrm{i}>$, where $<_{\mathrm{i}}$ is the 'i-part relation' and the sum operation applied to a nonempty set $X$ is defined as $\sup _{<i}(X)$. Given that individuals act as atoms of such a lattice, a different part relation would apply to them. Pluralities are mapped onto corresponding individuals in order to account for higher-order group formation with and. Distributivity for Link, moreover, consists in quantification over the i-parts of sums.

The conceptual resources available from Link's account are those of atom vs. proper sum and different part relations for different domains of entities. This is obviously too little to explain the facts discussed in this paper. The account would explain why distributivity is possible only with pluralities, not with groups (since it uses the i-part relation only). But the account does not provide a nonstipulative way of explaining why some predicates or readings of predicates apply only to sums and not atoms. Moreover, it does not provide a way of explaining why predicates of 
form have only a distributive reading, whereas predicates describing events display distributive and collective readings.

Landman (1989) gives an account of plurals which traces the availability of collective or distributive interpretations to the nature of the argument involved. For Landman, definite plural NPs denote sets of individuals if the predicate gets a distributive interpretation, and their denotation is lifted to the singleton of that set if the predicate receives a distributive interpretation. Thus, distributivity is not traced to the presence of a distributive operator (in the sentence meaning or the content of the predicate), but rather to the nature of the group argument.

This account clearly misses several generalizations this paper has established. First, it misses the generalization that not only distributivity, but also certain characterizable classes of collective predicates (or predicates on a collective reading) are possible only with plural arguments.

Both Link's and Landman's account, moreover, have a severe limitation, namely in that their account could not be carried over to the analogous behavior of kinds and properties.

\subsection{2. integrated wholes}

The approach I develop in Moltmann (1997) makes crucial use of the notion of integrated whole. An integrated whole, roughly, is an entity that has a boundary or shape, or displays integrity in some other way. Singular count nouns generally express properties of integrated wholes. By contrast, plurals and mass nouns don't. A plurality of objects, with more than one object, generally, is not an integrated whole and a quantity of water is not either. Only if the nonlinguistic context specifies relevant integrity conditions can a plurality of objects count, in the context, as an integrated whole. Predicates or readings of predicates that make reference to the parts but not the whole of an argument require the argument not to be an integrated whole in the context. For this reason, distributivity as well as the predicates in (35-40) are not applicable to integrated wholes.

The deficiencies of this account are twofold. First, the account does not explain why predicates of size or shape do not have a collective interpretation with pluralities. Second, the account is incapable of generalizing to properties and kinds. Integnity hardly applies to properties and thus properties and kinds cannot be distinguished on the basis of the notion of integrity. There are also problems with the notion of integrated whole when limited to particulars. A number of count nouns do not express any specific integnity conditions, for example thing, entity, quantity, or object. But these noums have the same effect of blocking distributivity and the application of certain predicates as singular count nouns expressing specific integrity conditions:

\section{John distinguished that entity. (no internal reading)}

In Moltmann (1997), I argued that these nouns impose implicit integrity conditions or merely conceived integrity. But of course the notion of a merely conceived integrated whole is quite problematic. On the present account, it is not integrity that plays the crucial role for objecthood and the application of the relevant predicates, but rather the ability to fulfil basic properties. Thus, the lexical content of object, 
quantity, or entity need not involve any sort of integrity, but rather only the general condition of being an object, i.e. a potential bearer of basic properties.

\section{Conclusion: Criteria for Objecthood}

In this paper, I have argued for an ontological criterion of objecthood — being a potential bearer of a basic property. This criterion coincides to a great extent with the linguistic criterion of being described by a singular count noun. One might even say the notion of object as a potential bearer of basic properties gives content to singular count nouns generally. However, the ontological criterion as such is independent of any linguistic notion such as a syntactic category.

Positing an ontological criterion for objecthood goes against a dominant tradition in philosophy, represented by Frege, Quine and others, which posits a linguistic criterion for objecthood instead. For Frege, to be an object is to be the referent of a referential noun phrase, and as such to be a possible argument of a predicate. For Quine, 'to be is to be the value of a variable'. 5 Given the present account, such linguistic criteria are misguided: they may identify entities or semantic objects, but there is yet another distinction to be made them that will yield the class of true objects.

\section{Appendix: Modeltheoretic semantics for a fragment of English}

In this appendix, I will sketch a direct model-theoretic semantics

for a fragment of English containing one- and two-place predicates, bare mass nouns and plurals, adjective nominalizations, and explicit property-referring terms (but no quantifiers and variables). The fragment will be disambiguated, though. Occurrences of nouns as predicates and as head nouns of NPs will be distinguished from referential occurrences of nouns as bare mass nouns and plurals. In the latter case, I will have the noun be subscripted by 'nom', as in children nom (the 'nominalization' of the noun child). 'nom' will also replace the various morphemes that form adjective nominalizations. Thus instead of wisdom, we will have wisenom.

I will assume that predicates denote properties as primitive objects. Such properties, however, will not act as objects in the object language (since they do not occur as arguments of predicates). The properties that are predicate denotations rather form the basis for the semantics of nominalizations, that is, the semantics of bare plurals and mass nouns as well as adjective nominalizations. This rather platonic assumption is not crucial, though. Alternatively, to satisfy a nominalist, the modeltheoretic structure could contain the predicates themselves.

A model $\mathrm{M}$ for the fragment of English is a structure $<<\mathbf{P}, \mathbf{R}, \mathbf{P}_{\text {int }}, \mathbf{R}_{\text {int }}>,<\mathbf{E}$, $\mathbf{N}, \mathbf{O}>,\left\langle\mathbf{I},<\mathrm{i}>,\left\langle\mathbf{M},<\mathrm{m}>, \mathbf{W}, \mathbf{T}, \mathrm{k}\right.\right.$, int, reif, corr, $\left.\left\langle\mathrm{D}_{1}, \mathrm{D}_{2}, \mathrm{D}_{3}, \mathrm{D} 4,\left\{\mathrm{D}^{\prime}, \mathrm{D}_{5}{ }^{\prime}, \ldots\right\}\right\rangle, \mathrm{F}\right\rangle$ with the following components:

(i) nonempty sets of properties $\mathbf{P}$ and (two-place) relations $\mathbf{R}$ (possible predicate denotations). $\mathbf{P}$ and $\mathbf{R}$ contain 'extensional' properties and relations in the sense that the extensions of those properties and relations consist in sets of entities or relations among entities. $\mathbf{P}_{\text {int }}$ and $\mathbf{R}_{\text {int }}$ are nonempty sets of intensional properties and 
relations, that is, properties and relations whose extension consists in intensions or relations between entities and intensions.

(ii) a nonempty set of entities $\mathbf{E}$, which divides into a subset $\mathbf{O}$ of objects and a subset $\mathbf{N}$ of nonobjects. $\mathbf{I}$ is another subset of of $\mathbf{E}$ consisting of individuals and pluralities. $I$ is ordered by a part relation $<i$ and is closed under sum formation sup $<$. (as in Link (1983) $<\mathbf{I},<_{i}>$ is a complete join semilattice.) The set $\mathbf{O}$ consists of the atoms of $\mathbf{I}$, whereas the nonatoms of $\mathbf{I}$ form a subset of $\mathbf{N}$. M contains two other complete join semilattices $\left\langle\mathbf{M},<_{\mathrm{m}}>\right.$ and $\left\langle\mathbf{K},<_{\mathrm{k}}\right\rangle$, where $\mathbf{M}$ (the set of quantities) is a subset of $\mathbf{N}$ and $<\mathrm{m}$ a part relation ordering it, and $\mathbf{K}$ (the set of kinds) also is a subset of $\mathbf{N}$ ordered by the part relation $<\mathrm{k}$.

(iii) a set of worlds $\mathbf{W}$ and a set of times $\mathbf{T}$

(iv) a partial function $\mathrm{k}$ mapping elements of $\mathbf{P}$ onto kinds, i.e., elements of $\mathbf{N}$.

(v) an intension function int. int maps properties in $\mathbf{P}, \mathbf{R}, \mathbf{P}_{\text {int }}$, or $\mathbf{R}_{\text {int }}$ onto intensions, functions mapping a world and time to an extension. Thus, for $P \in \mathbf{P}, w$ $\in \mathbf{W}$ and $t \in \mathbf{T}$, we will have int $(\mathrm{P})(\mathrm{w}, \mathrm{t}) \subseteq \mathbf{E}$, and for $\mathrm{P} \in \mathbf{P}_{\text {int }}$, int $(\mathrm{P})(\mathrm{w}, \mathrm{t})$ will be a set of functions either from WxT to $\mathbf{E}$ or from WxT to $\wp(\mathbf{E})$. int moreover maps kinds, elements of $\mathbf{K}$, onto intensions. The intension of the property $\mathrm{P}$ denoted by a (plural or mass) noun is the same as the intension of the kind denoted by the 'nominalization' of the noun (the bare plural or mass noun), i.e. $\operatorname{int}(\mathrm{P})=\operatorname{int}(\mathrm{k}(\mathrm{P})$ ). This identity does not hold for adjectives: the elements in the extension of wise are individuals, whereas the instances of (elements in the extension of) wisdom are tropes.

(vi) a reification function reif. reif maps elements of $\mathbf{N}$ onto elements of $\mathbf{O}$. (vii) a partial function of intensional correlate corr which maps 'extensional' properties or relations onto corresponding intensional properties or relations, that is, which maps some members of $\mathbf{P}$ or $\mathbf{R}$ onto members of $\mathbf{P}_{\text {int }}$ or $\mathbf{R}_{\text {int }}$.

(viii) five functions or sets of functions $\mathrm{D}_{1}, \mathrm{D}_{2}, \mathrm{D}_{3}, \mathrm{D}_{4},\left\{\mathrm{D}_{5}{ }^{\prime}, \mathrm{D}_{5}{ }^{\prime \prime}, \ldots\right\}$, which map properties onto (derived) properties. They are defined as follows (the way D1 and D2 would apply to relations is obvious and need not be given):

For $P \in P, D 1(P)=\lambda w t x y\left[\exists y^{\prime}\left(y^{\prime} \in \operatorname{int}(y)(w, t)<x, y^{\prime}>\in \operatorname{int}(P)(w, t)\right)\right]$

For $P \in P, D 2(P)=\lambda w t x\left[G n y^{\prime}\left(y^{\prime} \in \operatorname{int}(y)(w, t)<x, y^{\prime}>\in \operatorname{int}(P)(w, t)\right)\right]$

For $R \in \mathbf{R}, \mathrm{D} 3(\mathrm{R})=\lambda \mathrm{wtx}[\mathrm{x} \in \operatorname{int}(\operatorname{corr}(\mathrm{P}))(\mathrm{w}, \mathrm{t})]$

For $\mathrm{P} \in \mathrm{P}_{\text {int }}, \mathrm{D} 4(\mathrm{P})=\lambda w \mathrm{w} x[\mathrm{x} \in \operatorname{int}(\mathrm{P})(\mathrm{w}, \mathrm{t})]$

(I will refrain from defining the functions D5', D5", ... Some of them should yield the content of instance-distribution predicates. Others should give reanalyses of eventive collective predicates.)

With these functions, we can define the notions of derived and basic property:: $P$ is derived iff for some $D_{i}(i<6)$ and $P^{\prime} \in P, P=D_{i}\left(P^{\prime}\right) ; P$ is basic otherwise (viii) an interpretation function $F$ mapping basic expressions of English onto elements of $\mathbf{P}$ or constructs from $\mathbf{W}, \mathbf{T}$ and $\mathbf{E}$ in the following way: for a proper name $\mathrm{X}, \mathrm{F}(\mathrm{X})$ is a constant function from $\mathbf{W} \times \mathbf{T}$ to $\mathrm{O}$ for an intransitive verb, noun or adjective $X, F(X) \in P$ for an intransitive verb $X, F(X) \in P_{\text {int }}$ for a transitive extensional verb $X, F(X) \in \mathbf{R}$ for a transitive intensional verb $X, F(X) \in R_{\text {int }}$ 
For a model M, the semantics of the English fragment can now be given as follows, where [ ] is the valuation function and $\mathrm{w} \in \mathbf{W}$ and $t \in \mathbf{T}$ :

(i) For a proper name $\mathrm{N},[\mathrm{N}]^{\mathrm{M}}=\mathrm{F}(\mathrm{N})$.

For a noun, adjective, or verb $X,[X]^{M}=F(X)$.

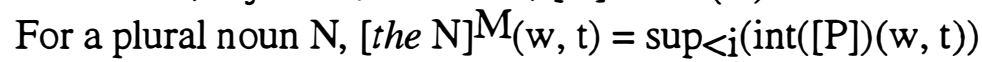

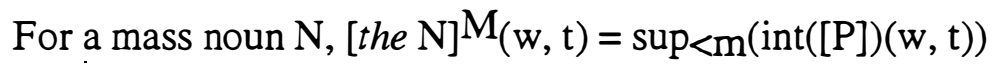

For a singular count noun $\mathrm{N},[$ the $\mathrm{N}] \mathrm{M}_{(\mathrm{w}, \mathrm{t})}=$ the object $\mathrm{o} \in \mathrm{O}$ such that $\mathrm{o} \in$ $\operatorname{int}([N])(w, t)$, if there exists such an object; undefined otherwise

For a plural or mass noun or adjective $X,\left[X_{n o m}\right]^{M}(w, t)=k([X])$

(ii) For a noun phrase NP, verb phrase VP, world $w$ and time $t$,

(1) if $[N P]^{M}(w, t) \in O$, then $[N P V P] M_{(w, t)}=1$ if $[N P]^{M}(w, t) \in$

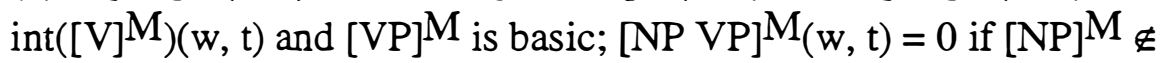
$\operatorname{int}\left([\mathrm{V}]^{\mathrm{M}}\right)(\mathrm{w}, \mathrm{t})$ and $[\mathrm{VP}]$ is basic; $\left[\mathrm{NP}\right.$ VP] ${ }^{\mathrm{M}}(\mathrm{w}, \mathrm{t})=$ undefined otherwise.

(2) if $[N P]^{M} \in N$, then $[N P V P]^{M}=1$ if $[N P]^{M} \in \operatorname{int}\left([V P]^{M}\right)(w, t)$ and [VP] $^{\mathrm{M}}$ is derived; [NP VP] ${ }^{\mathrm{M}}=0$ if $[\mathrm{NP}]^{\mathrm{M}} \notin \operatorname{int}\left([\mathrm{VP}]^{\mathrm{M}}\right)(\mathrm{w}, \mathrm{t})$ and [VP] ${ }^{\mathrm{M}}$ is derived; $[\mathrm{NP} V P]^{\mathrm{M}}(\mathrm{w}, \mathrm{t})=$ undefined otherwise

(iii) For a transitive verb $\mathrm{V},[\mathrm{V} N \mathrm{NP}]^{\mathrm{M}}=$ the property $\mathrm{P}, \mathrm{P} \in \mathbf{P}$ such that $\operatorname{int}\left(\mathrm{P}^{\prime}\right)(\mathrm{w}, \mathrm{t})=\lambda \mathrm{x}\left[<\mathrm{x},[\mathrm{NP}]^{\mathrm{M}}>\in \operatorname{int}(\mathrm{P})(\mathrm{w}, \mathrm{t})\right]$

Finally, the modeltheoretic meanings of some particular expressions:

(iv) For any $\mathrm{d} \in \mathbf{K}$, [property of](d) $=\operatorname{reif}(\mathrm{d})$.

For any $d$ such that for some $X \subseteq E,|X|>1, d=\sup _{<}(X)$,

[group of] $(\mathrm{d})=\left\{\mathrm{d}^{\prime} \mid \mathrm{d}^{\prime} \in \operatorname{int}(\mathrm{d})(\mathrm{w}, \mathrm{t}) \& \mathrm{~d}^{\prime}=\operatorname{reif}(\mathrm{d})\right\}$.

For any $\mathrm{d} \in \mathbf{M}$, [quantity of $](\mathrm{d})=\left\{\mathrm{d}^{\prime} \mid \mathrm{d}^{\prime} \in \operatorname{int}(\mathrm{d})(\mathrm{w}, \mathrm{t}) \& \mathrm{~d}^{\prime}=\operatorname{reif}(\mathrm{d})\right\}$.

\section{Endnotes}

${ }^{1}$ In what follows (mainly for reasons of space), I will adopt the judgment of those speakers, setting intuitions aside on which properties can be said to be rare or widespread.

2 For arguments that intensional verbs take properties as arguments, see Zimmerman (1992); for arguments that they take intensional quantifiers, see Moltmann (1997).

3 See also Moltmann (1997) for a detailed defense of that view.

4 A general analogy between universals and pluralities has also been argued for by Stout (1952).

5 For critical discussion of the syntactic criterion see also Wright (1983) and Hale (1987). 


\section{References}

Campbell, K. (1990): Abstract Particulars. Blackwell, Oxford.

Carlson, G. (1978): Reference to Kinds in English. PhD dissertation, University of Massachusets, Amherst.

Chierchia, G. (1998): 'Reference to kinds across languages'. Natural Language Semantics 6.4.

Chierchia, G. / R. Turner (1988): 'Semantics and Property Theory'. Linguistics and Philosophy 11.

Fine, K. (1982): Arbitrary Objects. Oxford UP, Oxford.

Grimshaw, J. (1979): 'Complement selection and the lexicon'. Linguistic Inquiry 10.

Hale, B. (1987): Abstract Objects. Blackwell, New York.

Landman, F. (1989): 'Groups I' and 'Groups II'. Linguistics and Philosophy 12.

Laurence, S. / C. Macdonald (eds.) (1998): Contemporary Readings in the Foundations of Metaphysics. Blackwell, Oxford.

Link. G. (1983): 'The logical analysis of plurals and mass nouns'. In R. Baeuerle et al. (eds.): Semantics from Different Points of View. de Gruyter, Berlin.

Lowe, E. J. (1998): The Possibility of Metaphysics. Substance, Identity, and Time. Clarendon Press, Oxford.

Moltmann, F. (1997a): Parts and Wholes in Semantics. Oxford UP, Oxford. Semantics 7.

Simons, P. (1994): 'Particulars in particular clothing: Three trope theories of substance'. Philosophy \& Phenomenological Research 54.3., reprinted in Laurence / Macdonald (eds.) (1998).

Stout, G. F. (1952): God and Nature. Cambridge UP, Cambridge.

Williams, D. C. (1953): 'On the elements of being'. Review of Metaphysics 7. Reprinted in Mellor/Oliver (eds.): Properties. Oxford UP, Oxford, 1997.

Wright, C. (1983): Frege's Conception of Numbers as Objects. Aberdeen UP, Aberdeen.

Zimmermann, E. (1992): 'On the proper treatment of opacity in certain verbs.' Natural Language Semantics 2. 